\title{
Beeinflusst die sinkende Wahlbeteiligung das Wahlergebnis? Eine Analyse kleinräumiger Wahldaten in deutschen Großstädten
}

\author{
Armin Schäfer
}

\section{Does Declining Voter Turnout Affect Election Results? An Analysis of Election Data from Districts in Major German Cities}

Abstract: Does declining voter turnout harm center-left parties? Although this is what many observers believe, most empirical studies fail to support this thesis. In contrast to this common wisdom, this article shows that there is a negative effect of turnout decline on the Social Democratic Party and the Left Party in Germany. For the first time, this article analyzes systematically aggregate data of 1,500 urban quarters in 34 large German cities. It will be argued that the effects of social segregation and voter apathy become only visible on this small scale since social indicators and turnout data correlate very highly. Urban districts differ not only in their electoral participation but also in the election results. Left of center parties fare much better in areas that have experienced the strongest decline of turnout over the last three decades.

Keywords: Turnout, Social Segregation, Center-left Parties, Aggregate Data, Contextual Effects

Schlagwörter: Wahlbeteiligung, soziale Segregation, Mitte-links-Parteien, Aggregatdaten, Kontexteffekte

\section{Einleitung ${ }^{1}$}

Bei der Bundestagswahl 2009 erreichte die Wahlbeteiligung einen neuen Tiefstand. Damit setzt sich auf Bundesebene ein Trend fort, der seit den Achtzigerjahren bei Landtags-, Kommunal- und Europawahlen zu beobachten ist: Immer weniger Menschen wählen. Doch hinter den Durchschnittswerten verbergen sich große Unterschiede in der Wahlbeteiligung (Schäfer 2011a): So hat bei der Bundestagswahl 2009 nur jeder zweite gewählt, der sich selbst der Unterschicht zurechnet, während dies 94 Prozent der Oberschichtangehörigen für sich reklamieren. Noch in den Siebziger- und Achtzigerjahren fiel die Kluft in der Wahlteilnahme deutlich geringer aus, doch seither ist sie stetig angewachsen. Ähnliche Unterschiede zeigen sich, wenn man auf die Verteilung der Wahlbeteiligung innerhalb von Großstädten blickt: Bei der Bundestagswahl lagen in Dresden 27

1 Frühere Fassungen dieses Aufsatzes wurden im Januar 2011 auf der Dreiländertagung in Basel und im Juni 2011 auf der Jahrestagung des Arbeitskreises „Wahlen und politische Einstellungen“ der DVPW vorgestellt. Für zahlreiche Hinweise danke ich Achim Goerres und den Tagungsteilnehmern sowie zwei anonymen Gutachtern. Außerdem danke ich den Mitarbeiterinnen und Mitarbeitern der städtischen Statistikbehörden, die nicht nur die Daten bereitstellten, sondern vielfach geduldig Nachfragen beantwortet haben. 
Prozentpunkte zwischen dem Stadtteil mit der höchsten und dem mit der niedrigsten Wahlbeteiligung, in Dortmund waren es 35, in Nürnberg 40 und Hamburg mehr als 45 Prozentpunkte. Auch hier ist der Abstand im Zeitverlauf größer geworden. Die ungleiche Wahlbeteiligung wirft die Frage auf, welche Auswirkungen sie auf den Wahlausgang hat.

Diese Frage wird in diesem Aufsatz beantwortet, indem eine in der Wahlforschung bislang selten genutzte Datenquelle erschlossen wird: Daten zur Wahlbeteiligung und den Wahlergebnissen bei Bundestagswahlen von etwa 1500 Stadtteilen in 34 deutschen Großstädten. Die Analyse von Stadtteildaten ermöglicht es, verhältnismäßig kleine und homogene Einheiten miteinander zu vergleichen. So bestehen beispielsweise die sechs Hamburger Bundestagswahlkreise aus insgesamt 100 Stadtteilen, zu denen Informationen über Wahlbeteiligung, Wahlergebnisse und soziale Lage vorliegen. Erst auf dieser kleinräumigen Ebene werden die Auswirkungen von sozialer Segregation und einer differenziellen Wahlbeteiligung auf den Wahlausgang sichtbar: Die Kenntnis des sozialen Profils von Stadtteilen ermöglicht zuverlässige Aussagen über das Abschneiden der Parteien. Dabei ergibt sich ein auf den ersten Blick verblüffendes Ergebnis: SPD und Die Linke erzielen ihre besten Ergebnisse dort, wo die Wahlbeteiligung niedrig ausfällt, während die CDU und insbesondere die FDP in Vierteln mit hoher Beteiligung am besten abschneiden. Gerade dieses Muster erklärt jedoch, weshalb der Rückgang der Wablbeteiligung linken Parteien schadet: In SPD-Hochburgen, so zeigt der Längsschnitt, ist die Wahlbeteiligung am stärksten zurückgegangen. Zwar schneidet die SPD in denselben Stadtteilen wie in der Vergangenheit überdurchschnittlich gut ab, aber bei einer sehr viel geringeren Wahlbeteiligung. Am Beispiel der Stadt Bremen lässt sich zeigen, dass für die elf Bundestagswahlen seit 1972 ein positiver Zusammenhang zwischen Wahlbeteiligung und dem SPD-Ergebnis für die Gesamtstadt besteht, während sich auf der disaggregierten Stadtteilebene ein im Zeitverlauf stärker werdender negativer Zusammenhang entwickelt.

Ausgangspunkt dieses Aufsatzes ist im nächsten Abschnitt ein Literaturüberblick zur Frage, ob Wahlergebnisse von der Höhe der Wahlbeteiligung beeinflusst werden. In der Forschung wird dies zumeist verneint, da sich die durch Umfragen ermittelten politischen Präferenzen von Wählern und Nichtwählern nicht stark unterscheiden. Anschließend werden im dritten Abschnitt die methodische Vorgehensweise und die Vorteile der Analyse von kleinräumigen Daten erläutert. Abschnitt vier zeigt, wie die soziale Lage von Stadtteilen mit der Wahlbeteiligung zusammenhängt. Am niedrigsten fällt die Wahlbeteiligung heute in „städtischen Problemgebieten “ (Falter u. Schumann 1994, S. 167) mit hoher Arbeitslosigkeit und hohem Migrantenanteil aus. Der fünfte Abschnitt benutzt Umfragedaten, um erste Hinweise zu sammeln, ob die Wohngegend einen von individuellen Merkmalen unabhängigen Effekt auf die Bereitschaft hat, wählen zu gehen. Da dies so ist, verstärkt die soziale Segregation Unterschiede in der Wahlbeteiligung sozialer Gruppen. Dies führt zur zentralen Frage, die im sechsten Abschnitt behandelt wird, ob sich eine sinkende Wablbeteiligung auf die Wahlergebnisse auswirkt. Im Schlussteil werden die Ergebnisse zusammengefasst und Anregungen für die weitere Forschung formuliert. 


\section{Literaturüberblick: Beeinflusst die Höhe der Wahlbeteiligung das Wahlergebnis?}

Bereits in den dreißiger Jahren des letzten Jahrhunderts formulierte Tingsten (1975 [1937], S. 230) das „Gesetz der Streuung “ (law of dispersion): Je niedriger die Wahlbeteiligung ist, desto ungleicher fällt die Wahlbeteiligung sozialer Schichten aus. Dieser Zusammenhang ist seitdem mehrfach im internationalen Vergleich bestätigt worden (Mahler 2008; Schäfer 2011b), sodass Kohler (2006, S. 170171) feststellt: „Niedrige Wahlbeteiligung ist generell ungleiche Wahlbeteiligung.“ Wenn nun angenommen wird, dass Geringgebildete und Einkommensschwache zu Parteien links der Mitte tendieren, sollte eine niedrige Wahlbeteiligung diesen Parteien schaden (Lijphart 1997, S. 4). Doch die vorliegenden empirischen Befunde sprechen in der Mehrzahl gegen diesen Zusammenhang. Die Herausgeber eines Sonderhefts zu diesem Thema resümieren den Forschungsstand wie folgt:

"The main finding of this volume is that turnout does not matter a great deal, no matter what method, dataset or period of time the authors apply. The various contributions each demonstrate that overall effects are minimal. Where they can be observed, they do not point in the originally expected direction that low turnout harms left-of-centre parties” (Lutz u. Marsh 2007, S. 544).

Wie im Folgenden argumentiert wird, könnte diese Schlussfolgerung vorschnell sein, da mit Umfragen die Nichtwähler möglicherweise nur unzureichend ermittelt werden können und Aggregatdatenanalysen häufig räumliche Einheiten untersuchen, die zu heterogen sind, um die Effekte einer differenziellen Wahlbeteiligung zu erfassen.

Arbeiten, die auf der Auswertung von Individualdaten beruhen, vergleichen in der Regel die Einstellungen und Parteineigungen von Wählern und Nichtwählern miteinander, um dann zu simulieren, wie sich eine höhere Wahlbeteiligung auf das Wahlergebnis auswirken würde. So gehen beispielsweise Highton und Wolfinger (2001) vor, die die Präsidentschaftswahlen 1992 und 1996 in den USA untersuchen. Zwar finden sie, dass die Nichtwähler der Demokratischen Partei näher stehen und eine größere Umverteilungsneigung aufweisen, aber die Unterschiede zu den Wählern sind so gering, dass auch eine höhere Wahlbeteiligung an den Wahlergebnissen nichts geändert hätte. Zum selben Ergebnis kommen Citrin et al. (2003) in ihrer Analyse amerikanischer Senatswahlen: Auch hier würde eine höhere Wahlbeteiligung in der Regel kein anderes Wahlergebnis herbeiführen, da sich die Präferenzen von Nichtwählern und Wählern ähneln. ${ }^{2}$ Dieselbe Vorgehensweise wird von van der Eijk et al. (2010) auf die Europawahl 2009 angewandt. $\mathrm{Da}$ die Wahlbeteiligung bei EP-Wahlen besonders niedrig ist, sollten sich bei ihnen klare Wahlbeteiligungseffekte zeigen. Doch die Autoren weisen nach, dass eine höhere Beteiligung keine oder nur sehr moderate Auswirkungen auf das Abschneiden der meisten zur Wahl stehenden Parteien gehabt hätte. Zudem fällt der

2 Hansford und Gomez (2010) instrumentieren die Variable Wahlbeteiligung durch das Wetter am Wahltag und kommen auf dieser Grundlage zu dem gegenteiligen Schluss, dass es in den USA einen positiven Effekt der Wahlbeteiligung auf das Abschneiden der Demokratischen Partei gibt. 
negative Effekt einer niedrigen Wahlbeteiligung für linke und libertäre Parteien in der Tendenz geringer als für rechte und autoritäre Parteien aus. Schließlich simuliert Kohler (2011) für alle Bundestagswahlen seit 1949, wie die Wahlergebnisse, die Zusammensetzung des Parlaments und damit die Koalitionsmehrheiten bei einer Wahlbeteiligung von 90-100 Prozent ausgesehen hätten. Nur bei einer Bundestagswahl hätte eine höhere Beteiligung mit hoher Wahrscheinlichkeit zu einem anderen Ergebnis geführt: Nach Kohlers Berechnungen wäre bei einer höheren Wahlbeteiligung die SPD 2005 stärkste Partei geworden und hätte somit die große Koalition mit der CDU führen können.

Die Ursache für die geringen Auswirkungen der Wahlbeteiligung auf das Wahlergebnis sehen diese Studien darin, dass sich Wähler und Nichtwähler in ihren politischen Präferenzen nicht stark unterscheiden. Für Deutschland argumentiert Kleinhenz (1995, S. 229-230), dass Nichtwähler überwiegend „Bürger der Mitte“ sind. Ihre Entscheidung, nicht zu wählen, hängt von situativen Faktoren wie etwa von der Mobilisierung durch die Parteien ab. Am ehesten weisen die Nichtwähler aus seiner Sicht eine größere Distanz zu den Volksparteien auf, wie auch Eilfort (1994, S. 334) bestätigt. Falter und Schumann (1993; 1994, S. 203-206) zeigen, dass Protestparteien (damals: Republikaner, PDS und Grüne) von den Nichtwählern mit größerer Sympathie als von den Wählern betrachtet werden. Die Nichtwähler sind häufiger als Wähler von den etablierten Parteien insgesamt enttäuscht und entscheiden sich zwischen Protest- und Nichtwahl. Ein systematischer Nachteil für die SPD lässt sich aus diesen Ergebnissen nicht ablesen. Das gilt für die Mehrzahl der Studien, die die politischen Präferenzen von Wählern und Nichtwählern vergleichen.

Der Nachteil dieser Forschungsstrategie ist, dass angenommen werden muss, die in Umfragen ermittelten Nichtwähler repräsentierten die tatsächlichen Nichtwähler. Dies ist aber zum einen nicht gegeben, wenn die Wahrscheinlichkeit, an einer Befragung teilzunehmen, positiv mit der Wahrscheinlichkeit zu wählen korreliert (Tourangeau et al. 2010). Gerade diejenigen, die zum harten Kern der Nichtwähler gehören, könnten sich der Befragung entziehen, sodass ihre politischen Präferenzen nicht erfasst werden (Clausen 1968-69, S. 592-593; Lavies 1973, S. 45). ${ }^{3}$ Zum anderen gibt ein Teil der Befragten sozial erwünschte Antworten, sodass die aus Umfragen ermittelte Wahlbeteiligung regelmäßig über der tatsächlichen liegt („overreporting “). ${ }^{4}$ Da die Bereitschaft, das Fernbleiben von der Wahlurne zuzugeben, gerade bei höher Gebildeten geringer ausgeprägt ist (Bernstein et al. 2001), sind sich Nichtwähler und Wähler in sozialer Hinsicht möglicherweise ähnlicher als es das erklärte Wahlverhalten nahelegt. Obwohl beide Verzerrungsquellen in entgegengesetzte Richtungen wirken, bleibt die Unsicherheit, ob Nichtwähler durch Umfragen adäquat erfasst werden können. Um diese Nachteile der Umfrageforschung zu vermeiden, versucht ein anderer Forschungsstrang, den Effekt der

3 Berinsky (2002, S. 285) weist zudem nach, dass die Wahrscheinlichkeit, in einer Umfrage einzelne Fragen nicht zu beantworten, mit höherem sozioökonomischen Status der Befragten abnimmt, sodass Umfrageergebnisse zu Lasten der sozial Schwachen verzerrt sein können: "In polls, as in other avenues of public expression, it is the voice of the disadvantaged that is muted."

4 Siehe hierzu die Diskussion bei Kleinhenz (1995, S. 75-80) sowie Caballero (2005, S. 334-336). 
Wahlbeteiligung durch die Analyse offizieller Wahldaten zu ermitteln. ${ }^{5}$ Im Mittelpunkt stehen dabei die Auswirkungen der Wahlbeteiligung auf Mitte-links-Parteien. So zeigt Radcliff (1994) für die Präsidentschaftswahlen zwischen 1928 und 1980, dass in den US-Bundesstaaten ein substanzieller Effekt der Wahlbeteiligung auf das Abschneiden der Demokratischen Kandidaten besteht. Diesen Befund bestätigen Pacek und Radcliff (1995) sowohl im internationalen Vergleich als auch im Vergleich der deutschen Bundesländer: Insbesondere Mitte-links-Parteien sind aus ihrer Sicht negativ von einer sinkenden Wahlbeteiligung betroffen. Allerdings werden diese Studien für ihr methodisches Vorgehen kritisiert. In seiner Replik auf Radcliff erwidert Erikson (1995), dass die Ergebnisse für die USA ausschließlich dadurch erklärt werden, dass in den Südstaaten unabhängig voneinander die Wahlbeteiligung und der Stimmenanteil der Demokraten gestiegen sind. Da er dies nicht beachte, sitze Radcliff einem Scheinzusammenhang auf. Auf dieselbe Gefahr, gleichgerichtete, aber voneinander unabhängige Trends als kausal miteinander verknüpft zu interpretieren, weist Fisher (2007) hin, der deshalb in seiner Analyse besonderen Wert darauf legt, ob Veränderungen der Wablbeteiligung auch zu Veränderungen der Ergebnisse von Mitte-links-Parteien führen. Fisher untersucht drei Wahlarten: Nationale Parlamentswahlen in 23 OECD-Ländern, Europawahlen und britische Parlamentswahlen. Auf dieser Grundlage findet er keine systematischen Auswirkungen der Wahlbeteiligung auf Stimmengewinne linker Parteien.

Viele Studien, die Aggregatdaten analysieren, vergleichen Länder, Bundesstaaten oder Wahlkreise miteinander. Für die USA haben Hajnal und Trounstine (2005) jedoch demonstriert, dass sich erst auf der Ebene von Stadtteilen die Auswirkungen einer niedrigen Wahlbeteiligung nachweisen lassen, weil nur in kleinräumigen Analysen der Effekt sozialer und ethnischer Segregation erkennbar wird. So führen Unterschiede in der Wahlbeteiligung ethnischer Gruppen bei Bürgermeisterwahlen in US-Städten dazu, dass hispanische oder asiatische Kandidaten geringere Erfolgschancen als ihre weißen Konkurrenten haben (umfassend hierzu Hajnal 2010). Diese Ergebnisse lassen vermuten, dass sich auch in Deutschland die Effekte einer fallenden Wahlbeteiligung vor allem in kleinräumigen Analysen und weniger durch die Analyse von Wahlkreis- oder Länderdaten zeigen, doch nur wenige Studien haben bislang den Blick auf Stadtteile gerichtet. So untersucht beispielsweise Geiling (2009), wie die SPD in Hannover in traditionellen Arbeitervierteln an Rückhalt und ehemalige Wähler an die Nichtwähler verloren hat, und Gardemin (2009) dokumentiert, dass sich die Hochburgen der Parteien sozialräumlich unterscheiden. Hennig et al. (2001) gehen am Beispiel von Frankfurt am Main der Frage nach, welche sozialräumlichen Variablen die Wahlenthaltung erklären können. Über mehrere Wahlen hinweg zeigt sich, dass Stadtteile stabil Nichtwähler- und Wählerhochburgen zuzurechnen sind. Während sich erstere in „Mehrproblemgebieten“, Arbeitervierteln und in der Frankfurter Innenstadt finden, zählen Mittelschichtgebiete und gehobene Wohngebiete zu letzteren. Ganz ähnliche Befunde werden für Bremen berichtet, wo sich eine „be-

5 Zur Diskussion der Vor- und Nachteile von Aggregatdatenanalyse siehe Schoen (2005, S. 94-101). 
schleunigende Erosion der Wahlbeteiligung in den Wählermilieus der sozial prekären Ortsteile" beobachten lässt (Schlichting 2009, S. 62).

Auch die Wahlerfolge rechter Parteien Anfang der Neunzigerjahre gaben Anlass zu ermitteln, in welchen Stadtvierteln sie besonders stark vertreten waren. So analysieren Jagodzinski et al. (1996) auf Stadtteilebene den Zusammenhang zwischen sozialen Indikatoren wie Bildung und Arbeitslosigkeit einerseits und dem Erfolg von Republikanern und DVU anderseits. Während rechte Parteien in Hamburg und Berlin in jenen Stadtteilen gut abschnitten, wo die Wahlbeteiligung niedrig und der Anteil der Bewohner mit Hauptschulabschluss hoch war, erzielten die Grünen ihre Erfolge in Stadtteilen mit hoher Wahlbeteiligung und einem hohen Abiturientenanteil. Die Kenntnis des sozialen Profils von Stadtteilen ermöglicht relativ zuverlässige Aussagen sowohl über die Wahlbeteiligung als auch über das Abschneiden der Parteien. Im Folgenden wird daher eine erste umfassende Analyse von kleinräumigen Daten deutscher Großstädte vorgenommen, die über Fallstudien zu einzelnen Städten hinausgeht, um der Frage nachzugehen, ob ein Zusammenhang zwischen der (Veränderung der) Wahlbeteiligung und den Wahlergebnissen besteht.

\section{Daten und Methode}

Offizielle Wahldaten sind im Unterschied zu Umfragedaten genau. Probleme mit sozial erwünschten Angaben zum Wahlverhalten treten nicht auf. Der Nachteil von Daten zur räumlichen Verteilung von Wählern und Nichtwählern liegt jedoch darin, dass nur selten ausreichend Indikatoren vorliegen, mit deren Hilfe die Wahlbeteiligung erklärt werden kann. Auch der Schluss von Makrodaten auf Individuen ist problematisch (Robinson 1950), weshalb die nachfolgenden Analysen Zusammenhänge auf der Makroebene beschreiben. Wenn Aggregatdaten in der deutschen Wahlforschung analysiert werden, sind dies entweder die Wahlergebnisse in den Wahlkreisen, da für diese eine Reihe von Indikatoren vom Bundeswahlleiter bereitgestellt werden (z. B. Schoof 1981; Falter u. Schumann 1994), oder Daten aus der Regionalstatistik, die eine Fülle von Informationen zu den ca. 600 Kreisen und kreisfreien Städten in Deutschland beinhaltet (Steinbrecher et al. 2007). Noch kleinteiligere Daten zur Wahlbeteiligung, aber auch zur Sozialstruktur in den Stadtteilen stellen die Statistikämter vieler Großstädte bereit.

Da größere räumliche Einheiten zumeist intern heterogen sind, erhöhen Daten zu kleineren und homogeneren Einheiten die Varianz. So lagen bei der Bundestagswahl 2009 zwischen der höchsten und niedrigsten Wahlbeteiligung in einem Wahlkreis 22 Prozentpunkte, während in Städten wie Hamburg oder Köln fast 50 Prozentpunkte zwischen der Wahlbeteiligung in den Stadtteilen liegen. Auch Indikatoren wie die Arbeitslosenquote oder das Einkommen streuen in Großstädten auf engstem Raum. Diese Informationen gehen verloren, wenn Wahlkreise analysiert werden. So umfasst beispielsweise der Wahlkreis 96 im Kölner Nordwesten sowohl den Stadtteil Esch-Auweiler, der bei Armut, Arbeitslosigkeit und Migrantenanteil unter dem Kölner Durchschnitt liegt, als auch den Stadtteil Chorweiler, der weit überdurchschnittliche Werte aufweist. Zwischen diesen beiden Stadtteilen desselben Wahlkreises lagen bei der Bundestagswahl 2009 mehr als 30 Pro- 
zentpunkte in der Wahlbeteiligung. Greift man auf kleinräumige Stadtteildaten zu, liegen pro Wahlkreis bis zu 20 Datenpunkte vor, sodass sich manche Zusammenhänge klarer zeigen.

Für die folgenden Analysen wurden Daten von 34 Großstädten zur Wahlbeteiligung und zum Wahlergebnis in einer Datenbank zusammengefasst. Insgesamt gab es 200938 Großstädte mit mehr als 200.000 Einwohnern in Deutschland, doch nur für 34 davon liegen Informationen zur Wahlbeteiligung auf Stadt- oder Ortsteilebene vor. ${ }^{6}$ Für manche Städte sind Daten für die Wahlkreise der Kommunalwahlen (Berlin: Abgeordnetenhauswahl), nicht aber Informationen auf Stadtteilebene verfügbar. In allen Fällen war es das Ziel, Informationen der kleinsten verfügbaren räumlichen Einheit zu sammeln. Der Einfachheit halber wird im Folgenden dennoch durchgängig von Stadtteilen oder Vierteln gesprochen. Insgesamt werden Wahldaten von 1494 Stadtteilen verwendet. Für manche Analysen kann jedoch nur eine Untergruppe der Städte analysiert werden. So sind nur für 749 Stadtteile Daten zur Arbeitslosenquote oder zur Arbeitslosendichte vorhanden. Da hinsichtlich der sozialen Lage nur wenige Indikatoren für mehrere Städte existieren, wird die Querschnittanalyse durch eine detaillierte Betrachtung einzelner Städte - insbesondere Bremen, Duisburg, Hamburg und Köln - ergänzt.

Während die Auswertung von Stadtteildaten kleinräumige Analysen mit offiziellen Daten ermöglicht, sind zum Teil keine multivariaten Regressionsanalysen möglich, da zu wenige Variablen vorliegen. Daher werden im Folgenden überwiegend bivariate Korrelationen und Streudiagramme dargestellt. Dies birgt die Gefahr, dass die Zusammenhänge durch nicht beobachtete Drittvariablen erklärt werden könnten. Wo immer es möglich ist, werden die Nachteile der Korrelationsanalyse durch ergänzende multivariate Analysen ausgeglichen. Ein weiteres methodisches Problem besteht darin, dass sich die Größe der Stadtteile zwischen den Städten unterscheidet. Da jedoch räumliche Einheiten mit weniger Einwohnern tendenziell homogener sind, können diese Unterschiede die Auswertung verzerren. Beispielsweise gibt es in München nur 25 Stadtteile mit durchschnittlich 35.000 Wahlberechtigten, während Nürnberg Daten für 80 Stadtteile mit durchschnittlich nur etwa 4000 Wahlberechtigten bereitstellt. Das heißt, die Stadtteile beider Städte sind nicht direkt miteinander vergleichbar, weil die Münchener Daten heterogenere Einheiten umfassen. Um dieses Problem zu umgehen, werden in einigen Analysen die Variablen z-transformiert, sodass sie für jede Stadt einen Mittelwert von 0 und eine Standardabweichung von 1 haben. Die Aussagen der empirischen Untersuchung beziehen sich in diesen Fällen darauf, wie sich die Abweichung der Wahlbeteiligung eines Stadtteils vom Durchschnitt der gesamten

6 Die Städte sind Aachen, Augsburg, Berlin, Bielefeld, Bochum, Bonn, Braunschweig, Bremen, Chemnitz, Dortmund, Dresden, Duisburg, Düsseldorf, Erfurt, Essen, Frankfurt a. M., Freiburg, Gelsenkirchen, Halle (Saale), Hamburg, Hannover, Köln, Krefeld, Leipzig, Lübeck, Mannheim, München, Münster, Nürnberg, Oberhausen, Rostock, Stuttgart, Wiesbaden und Wuppertal. Insgesamt haben diese 34 Städte knapp 19 Million Einwohner (Daten: Statistische Ämter des Bundes und der Länder, Stand: 6.12.2010). 
Stadt auf die Abweichung des Parteiergebnisses vom Durchschnittsergebnis auswirkt. $^{7}$

\section{Unterschiede in der Wahlbeteiligung zwischen Stadtteilen}

Die Wahlbeteiligung innerhalb von Großstädten hängt eng mit der soziale Lage der einzelnen Stadtteile zusammen. In Stadtvierteln mit überdurchschnittlichem Einkommensniveau, geringer Arbeitslosigkeit und geringerem Migrantenanteil liegt die Wahlbeteiligung regelmäßig über dem Durchschnitt der Gesamtstadt, während sie in von Arbeitslosigkeit und Armut stärker betroffenen Vierteln darunter liegt. Im Folgenden wird die soziale Lage entweder durch die Arbeitslosequote erfasst oder durch den Anteil von Personen, die „Grundsicherung für Arbeitssuchende" erhalten (SGB II-Empfängerquote). ${ }^{8}$ Abbildung 1 stellt für 100 Hamburger Stadtteile den Zusammenhang zwischen der SGB II-Quote und der Wahlbeteiligung bei vier Abstimmungen dar. In allen Fällen besteht ein enger $\mathrm{Zu}$ sammenhang zwischen beiden Indikatoren: Je größer der Anteil von Hartz-IVEmpfängern in einem Hamburger Stadtteil, desto niedriger fällt dort die Wahlbeteiligung aus. ${ }^{9}$ Die Unterschiede zwischen der höchsten und der niedrigsten Wahlbeteiligung in einem Stadtteil betragen bei der EP-Wahl 42, bei der Bundestagswahl 47, beim Volksentscheid über die Schulreform 48 und bei der Bürgerschaftswahl 52 Prozentpunkte. Dasselbe Muster zwischen sozialer Lage, diesmal gemessen an der Arbeitslosenquote in den Stadtvierteln, und der Wahlbeteiligung tritt in allen 15 Städten auf, für die diese Daten verfügbar sind. ${ }^{10}$ Der Korrelationskoeffizient liegt zwischen -0,81 (München) und -0,98 (Essen) und ist in allen Fällen hoch statistisch signifikant. Fasst man die Datenpunkte der 710 Stadtteile dieser 14 Städte in einem Streudiagramm zusammen, wird die Beziehung zwischen Arbeitslosenquote und Wahlbeteiligung ersichtlich: Ist ein Stadtteil überdurchschnittlich von Arbeitslosigkeit betroffen, liegt die Wahlbeteiligung unter dem Durchschnitt (Abbildung 2). ${ }^{11}$

7 In den empirischen Analysen wurde, wann immer es möglich war, überprüft, ob der Einschluss der Stadtteilgröße, gemessen an der Bevölkerung oder an der Anzahl der Wahlberechtigten, einen Einfluss auf die dargestellten Zusammenhänge hat. Dies ist jedoch nicht der Fall, sodass alle Modelle ohne die Kontrollvariablen „Einwohnerzahl“ oder „Anzahl der Wahlberechtigten“ dargestellt werden.

8 Diese beiden Indikatoren stehen stellvertretend für eine Fülle weiterer Variablen, die stattdessen hätten verwendet werden können: das Durchschnittseinkommen, der Gymnasiastenanteil unter Jugendlichen, der Ausländeranteil, aber auch das Durchschnittsalter von Frauen bei der Geburt des ersten Kinds. Alle diese Indikatoren korrelieren hoch miteinander und verweisen gemeinsam darauf, dass deutsche Großstädte sozial und ethnisch segregiert sind (siehe Farwick 2007; Friedrichs u. Triemer 2008).

9 In allen vier Fällen ist der Korrelationskoeffizient statistisch signifikant und größer als 0,8.

10 Die 15 Städte sind Augsburg, Bielefeld, Bremen, Chemnitz, Düsseldorf, Erfurt, Essen, Frankfurt, Freiburg, Halle (Saale), Hamburg, Hannover, Köln, München und Wiesbaden.

11 Aufgrund der durchgängig negativen Korrelation zwischen der Arbeitslosenquote eines Stadtteils und der Wahlbeteiligung zu folgern, dass vor allem Arbeitslose nicht wählen, hieße jedoch, dem „ökologischen Fehlschluss“ (Robinson 1950) zu erliegen, wie Schoen (2005, S. 97-99) am Beispiel von Erwerbslosigkeit und Wahlbeteiligung erläutert. 
Abbildung 1: Wahlbeteiligung bei vier Abstimmungen in 100 Hamburger Stadtteilen

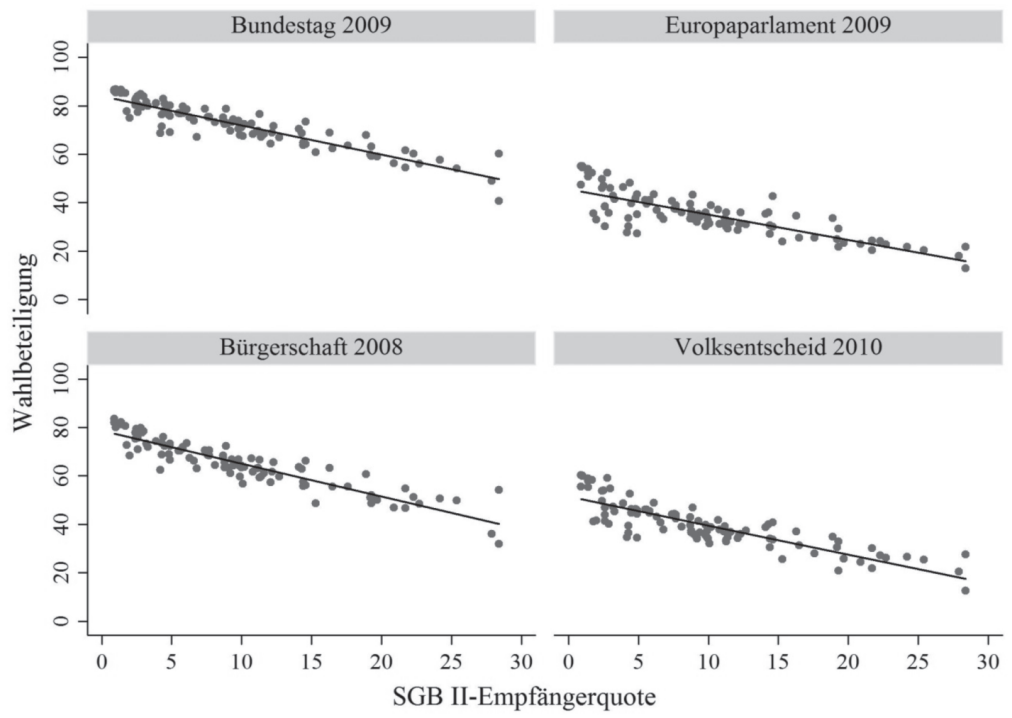

Quelle: Eigene Zusammenstellung aus Daten des Statistikamts Nord, Hamburg.

Abbildung 2: Arbeitslosenquote und Wahlbeteiligung in 710 Stadtteilen deutscher Großstädte

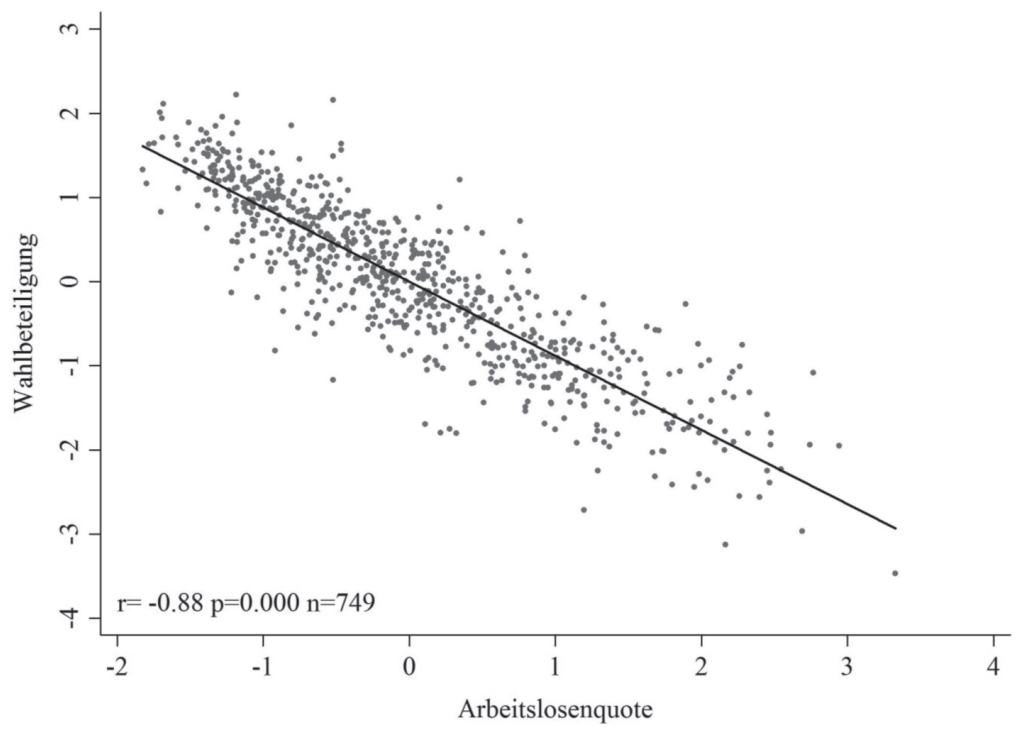

Anmerkung: Die Variablen sind für jede Stadt z-transformiert, sodass der Mittelwert o und die Standardabweichung 1 beträgt.

Quelle: Eigene Zusammenstellung auf Grundlage der Informationen der Statistikämter. 
Dieser Zusammenhang könnte jedoch darauf zurückgehen, dass in Vierteln mit hoher Arbeitslosenquote mehr Migranten mit Wahlrecht leben, die jedoch eine geringere Wahlbeteiligung aufweisen. Weniger die soziale, als vielmehr die ethnische Segregation wäre in diesem Fall für Unterschiede in der Wahlbeteiligung verantwortlich. Nur wenige Städte weisen die Migrantenanteile auf Stadtteilebene aus und unterscheiden zudem zwischen nicht-wahlberechtigten Ausländern und wahlberechtigten Migranten. Das Statistische Landesamt Bremen stellt diese Daten jedoch zur Verfügung, sodass zumindest exemplarisch überprüft werden kann, ob der Zusammenhang zwischen Arbeitslosigkeit und Wahlbeteiligung bestehen bleibt, wenn für den Anteil der wahlberechtigten Migranten kontrolliert wird.

Tabelle 1: Regressionsmodelle zur Wahlbeteiligung in 83 Bremer Stadtteilen

\begin{tabular}{lccc}
\hline AV: Wahlbeteiligung & Modell 1 & Modell 2 & Modell 3 \\
\hline Arbeitslose & $-1,117^{* * *}$ & $-0,665^{* * *}$ & $-0,501^{* * *}$ \\
Einkommen_2004 & $(0,0688)$ & $(0,105)$ & $(0,0814)$ \\
& & 0,122 & $0,158^{* *}$ \\
Abitur & & $(0,0655)$ & $(0,0493)$ \\
& & $0,160^{* *}$ & $0,122^{* * *}$ \\
Migranten & $(0,0470)$ & $(0,0355)$ \\
& & & $-0,364^{* * *}$ \\
Konstante & & & $(0,0468)$ \\
& $87,45^{* * *}$ & $70,70^{* * *}$ & $74,02^{* * *}$ \\
\hline $\mathrm{R}^{2}$ & $(1,069)$ & $(3,243)$ & $(2,466)$ \\
$N$ & 0,765 & 0,832 & 0,907 \\
\hline
\end{tabular}

Anmerkungen: ${ }^{*} \mathrm{p}<0,05,{ }^{* *} \mathrm{p}<0,01,{ }^{* * *} \mathrm{p}<0,001$. Arbeitslose: Anteil der Arbeitslosen an den sozialversicherungspflichtig Beschäftigten zuzüglich Arbeitslose; Einkommen_2004: Durchschnittliches Einkommen der Lohn- und Einkommensteuerpflichtigen in 1.000 EUR; Abitur: Anteil der Schüler an Gymnasien an der Bevölkerung im Alter von 16 bis unter 19 Jahren. Migranten: Anteil der Migranten minus Anteil der Ausländer.

Quelle: Statistisches Landesamt Bremen 2009.

In Tabelle 1 sind die Koeffizienten von drei Regressionsmodellen abgetragen, die die Höhe der Wahlbeteiligung in den Bremer Stadtteilen schätzen. Im ersten Schritt wird lediglich die Arbeitslosenquote als unabhängige Variable eingefügt. Mit jedem Prozentpunkt, die sie in einem Stadtteil höher liegt, sinkt die Wahlbeteiligung bei der Bundestagswahl 2009 um 1,1 Prozentpunkte. Im zweiten Schritt werden das Durchschnittseinkommen sowie der Gymnasiastenanteil aufgenommen. ${ }^{12}$ Während der negative Effekt der Arbeitslosenquote bestehen bleibt, hat nur die Bildungsvariable einen signifikanten, positiven Effekt. Schließlich wird im dritten Schritt der Anteil der wahlberechtigten Migranten in den Stadtteilen berücksichtigt. Und obwohl gilt, dass je größer der Anteil wahlberechtigter Migran-

12 Da es einen engen Zusammenhang zwischen dem Bildungsgrad von Eltern und Kindern gibt, dient diese Variable als Indikator für den durchschnittlichen Bildungsstand in einem Stadtteil. 
ten, desto niedriger die Wahlbeteiligung ist, bleibt die Arbeitslosenquote statistisch signifikant. ${ }^{13}$ Das heißt, unter sonst gleichen Bedingungen liegt die Wahlbeteiligung im Stadtteil mit der höchsten Arbeitslosenquote 14,5 Prozentpunkte niedriger als im Stadtteil mit der geringsten Quote.

Nun ließe sich vermuten, dass schon immer Unterschiede in der Wahlbeteiligung bestanden haben und dass der Rückgang der Wahlbeteiligung in allen Stadtteilen gleichmäßig verlaufen ist. Wie sich Abbildung 3 entnehmen lässt, ist dies jedoch nicht der Fall: Mit rückläufiger Wahlbeteiligung nimmt der Abstand zwischen der niedrigsten und der höchsten Wahlbeteiligung in einem Stadtteil zu. Trennten 198722 Prozentpunkte den Kölner Stadtteil mit der höchsten Beteiligung von dem mit der niedrigsten, waren es 200043 Prozentpunkte. Dasselbe Muster zeigt sich in Bremen (1972-2009) und Duisburg (1990-2009). Diese Daten bestätigen Tingstens „Gesetz der Streuung “: Mit sinkender durchschnittlicher Wahlbeteiligung nehmen die Beteiligungsunterschiede zu.

Abbildung 3: Nichtwähleranteil und Streuung der Wahlbeteiligung bei Bundestagswahlen in drei Städten

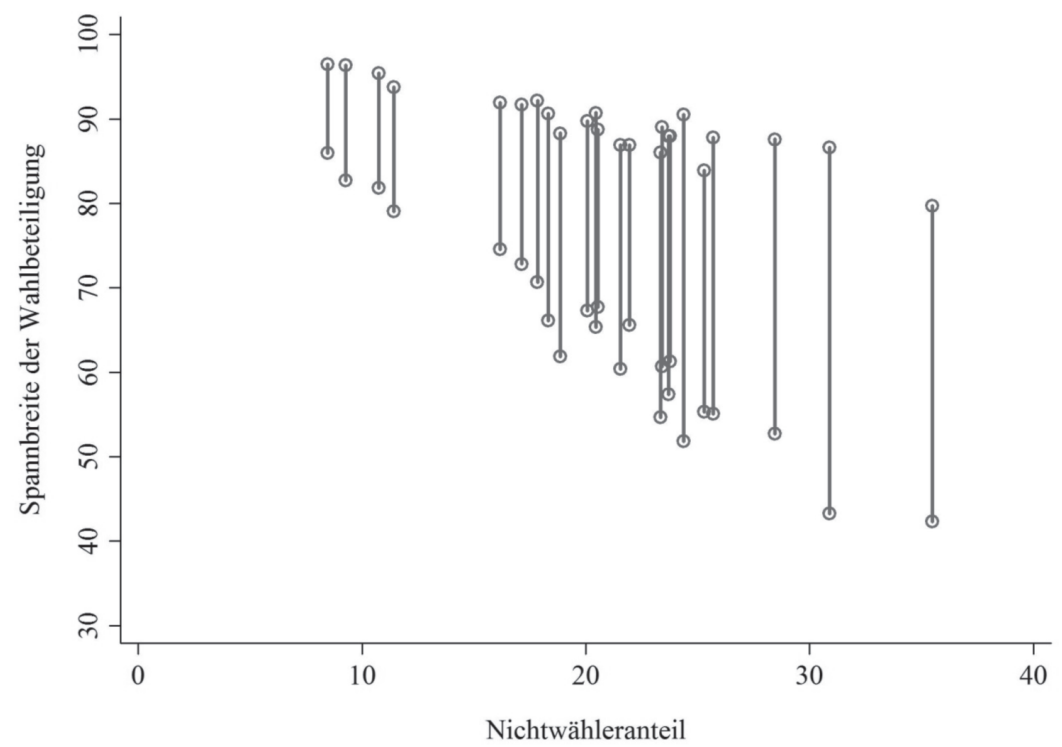

Anmerkung: Die Städte sind Bremen (1972-2009), Duisburg (1990-2009) und Köln (1987-2009).

Quelle: Statistisches Landesamt Bremen, Stabsstelle für Wahlen, Europaangelegenheiten und Informationslogistik der Stadt Duisburg sowie Amt für Stadtentwicklung und Statistik der Stadt Köln.

13 Trotz der starken Korrelation der unabhängigen Variablen verweisen Werte von unter fünf des Variance Inflation Factors darauf, dass Multikollinearität die Ergebnisse nicht verzerrt. Zudem zeigt der Breusch-Pagan-Test, dass keine Heteroskedastizität vorliegt. 
Bevor wir uns der Kernfrage zuwenden, wie sich der Rückgang der Wahlbeteiligung auf das Wahlergebnis auswirkt, wird im nächsten Abschnitt gefragt, ob Unterschiede zwischen Stadtteilen ausschließlich auf deren soziale Zusammensetzung zurückzuführen sind oder ob die Konzentration von Nichtwählern einen von individuellen Merkmalen unabhängigen, zusätzlichen Effekt auf die Wahlwahrscheinlichkeit der dort Lebenden ausübt.

\section{Kompositions- oder Kontexteffekte: Ergänzende Analysen auf Individualebene}

Der letzte Abschnitt hat gezeigt, dass soziale Lage und Wahlbeteiligung hoch korrelieren. Für die Unterschiede zwischen den Stadtteilen könnten zwei Mechanismen verantwortlich sein. Zum einen könnten sie schlicht die Zusammensetzung der Bevölkerung widerspiegeln. Je mehr Menschen mit geringer Wahlneigung in einem Viertel leben, desto niedriger ist die Wahlbeteiligung. Soziale Segregation führt in diesem Fall zur Konzentration von Nichtwählern in einzelnen Vierteln, übt aber keinen Einfluss auf die Beteiligungswahrscheinlichkeit der dort Lebenden aus. Falls jedoch die ungleiche Verteilung von Nichtwählern in einer Stadt die individuelle Bereitschaft verändert, die Stimme abzugeben, spricht man von einem Kontexteffekt. Dass die soziale Umgebung das individuelle Verhalten beeinflusst, ist umso wahrscheinlicher, je größer die „regionale Klumpung“ von Merkmalen ist (Pappi 1977, S. 157). Für deutsche Großstädte, die im Zentrum dieser Untersuchung stehen, trifft dies wie wir gesehen haben zu. Dennoch kann mit den bislang genutzten Daten die Frage nicht beantwortet werden, ob Kontexteffekte vorliegen. Hierzu wäre im Idealfall eine Kombination von Informationen auf individueller und auf Stadtteilebene notwendig. 
Tabelle 2: Wahlabsicht Kommunalwahl in Duisburg 2004

\begin{tabular}{|c|c|c|}
\hline $\begin{array}{l}\text { AV: Wahlabsicht }(1=\text { bestimmt } \rightarrow \text { wahrscheinlich; } \\
0=\text { bestimmt nicht } \rightarrow \text { vielleicht })\end{array}$ & Modell 1 & Modell 2 \\
\hline Alter & $\begin{array}{r}0,079^{*} \\
(0,035)\end{array}$ & $\begin{array}{c}0,063 \\
(0,040)\end{array}$ \\
\hline Alter ${ }^{2}$ & $\begin{array}{l}-0,001 \\
(0,000)\end{array}$ & $\begin{array}{l}-0,000 \\
(0,000)\end{array}$ \\
\hline $\begin{array}{l}\text { Geschlecht } \\
\text { (1=weiblich) }\end{array}$ & $\begin{array}{l}-0,474^{*} \\
(0,203)\end{array}$ & $\begin{array}{l}-0,182 \\
(0,239)\end{array}$ \\
\hline $\begin{array}{l}\text { in Partnerschaft lebend } \\
(1=\mathrm{ja} ; 0=\text { nein })\end{array}$ & $\begin{array}{c}0,085 \\
(0,219)\end{array}$ & $\begin{array}{c}0,164 \\
(0,241)\end{array}$ \\
\hline $\begin{array}{l}\text { arbeitslos } \\
(1=\mathrm{ja} ; 0=\text { nein })\end{array}$ & $\begin{array}{l}-0,219 \\
(0,244)\end{array}$ & $\begin{array}{l}-0,022 \\
(0,275)\end{array}$ \\
\hline \multicolumn{3}{|l|}{ Wähleranteil im Stadtteil ( $R K=$ sehr wenige) } \\
\hline durchschnittlich & $\begin{array}{l}0,456^{*} \\
(0,215)\end{array}$ & $\begin{array}{l}0,696^{* *} \\
(0,239)\end{array}$ \\
\hline hoch & $\begin{array}{l}0,671^{*} \\
(0,331)\end{array}$ & $\begin{array}{l}0,793^{*} \\
(0,373)\end{array}$ \\
\hline \multicolumn{3}{|l|}{ Bildung $(R K=$ böchstens Hauptschule $)$} \\
\hline mittlere Reife & & $\begin{array}{l}0,175 \\
(0,279)\end{array}$ \\
\hline mind. Fachhochschulreife & & $\begin{array}{l}0,915 * * \\
(0,316)\end{array}$ \\
\hline $\begin{array}{l}\text { Kirchgang } \\
(1=\text { nie } \rightarrow 5 \text { häufig })\end{array}$ & & $\begin{array}{l}0,202 * \\
(0,085)\end{array}$ \\
\hline $\begin{array}{l}\text { Gewerkschaftsmitglied } \\
(1=\mathrm{ja} ; 0=\text { nein })\end{array}$ & & $\begin{array}{c}0,326 \\
(0,293)\end{array}$ \\
\hline $\begin{array}{l}\text { politisches Interesse } \\
\text { (1=überhaupt nicht } \rightarrow 5=\text { sehr stark) }\end{array}$ & & $\begin{array}{l}0,725^{* * *} \\
(0,124)\end{array}$ \\
\hline Konstante & $\begin{array}{l}-0,422 \\
(0,763)\end{array}$ & $\begin{array}{l}-3,417 * \cdots * \\
(0,947)\end{array}$ \\
\hline pseudo $R^{2}$ & 0,043 & 0,166 \\
\hline$N$ & 891 & 848 \\
\hline
\end{tabular}

Anmerkungen: Standardfehler in Klammern; ${ }^{*} p<, 05,{ }^{* *} p<, 01,{ }^{* * *} p<, 001$.

Quelle: ZA-Nr. 4271, Schmitt-Beck, Mackenrodt und Faas.

Eine Annäherung an die Frage nach möglichen Kontexteffekten erlaubt eine Befragung zur Kommunalwahl 2004 in Duisburg (siehe hierzu: Schmitt-Beck et al. 2008). ${ }^{14}$ Neben einer Fülle von Fragen zum politischen Interesse und zur Neigung, über Politik zu diskutieren, wird in dieser Erhebung ebenfalls die Einschätzung abgefragt, wie viele Bürger im eigenen Stadtteil wählen („relativ wenige“, „durchschnittlich viele“, „sehr viele“). Falls das eigene Verhalten durch das Ver-

14 Die Daten sind unter der Nummer ZA-4271 bei der GESIS hinterlegt. Ich danke Rüdiger SchmittBeck dafür, mir den Datensatz zur Sekundäranalyse überlassen zu haben. 
halten anderer Bewohner eines Stadtteils geprägt wird, könnte diese Einschätzung die individuelle Wahlabsicht beeinflussen. Die Ergebnisse der statistischen Auswertung in Tabelle 2 bestätigen dies: Selbst unter Kontrolle anderer Erklärungsfaktoren wie Alter, Bildung und politischem Interesse verändert die Einschätzung, wie viele Wähler in einem Stadtviertel wohnen, die individuelle Wahlwahrscheinlichkeit. Substanziell bedeutet dies, dass sich die Wahlwahrscheinlichkeit einer Durchschnittsbefragten (bei der alle anderen Variablen mittlere Werte annehmen) um sechs Prozentpunkte erhöht, wenn sie angibt, in einem Viertel mit „sehr vielen“ statt in einer Gegend mit „relativ wenigen“ Wählern zu leben.

Tabelle 3: Wohnumgebung und soziale Lage

\begin{tabular}{lccccc}
\hline Wohnumgebung & $\begin{array}{c}\text { Haushalts- } \\
\text { einkommen }\end{array}$ & Bildung & Migranten & Arbeitslose & Wähler \\
\hline sehr schlecht bis & 1.294 & 25 & 26 & 11 & 62 \\
durchschnittlich & 1.633 & 29 & 13 & 5 & 81 \\
gut & 1.943 & 37 & 12 & 2 & 87 \\
sehr gut & &
\end{tabular}

Anmerkungen: Wohnumgebung: Vom Interviewer vorgenommene Bewertung der Wohngegend der Befragten; Haushaltseinkommen: Nach Personenzahl gewichtetes monatliches Haushaltsnettoeinkommen in Euro; Bildung: Anteil der Befragten, die mindestens Fachhochschulreife erreicht haben; Migranten: Anteil der nicht in Deutschland geborenen Befragten; Arbeitslose: Anteil der arbeitslosen Befragten; Wähler: Anteil der Wähler bei der Bundestagswahl 2009.

Quelle: ZA-Nr: 4610, ALLBUS 2010.

Um diesen ersten Befund abzusichern, wird zusätzlich die ALLBUS-Erhebung aus dem Jahr 2010 ausgewertet. Zwar lassen sich daraus keine detaillierten Angaben zum Wohnort der Befragten ermitteln, aber die Interviewer nehmen eine Bewertung der Wohnumgebung der Befragten vor (fünfstufig von „sehr schlecht“ bis „sehr gut“). Da die Wohnumgebung nur selten als „sehr schlecht“ oder "schlecht“ eingestuft wird, werden diese beiden Kategorien für die folgenden Analysen mit der mittleren Kategorie zusammengefasst, sodass „höchstens durchschnittliche“, „gute“ und „sehr gute“ Wohngegenden verglichen werden. Wie sich Tabelle 3 entnehmen lässt, teilt selbst diese grobe Unterscheidung die Befragten in klar getrennte Gruppen auf. Das verfügbare Haushaltseinkommen und der Bildungsgrad sind bei Befragten aus guten oder sehr guten Wohngegenden deutlich höher, während der Migrantenanteil und die Arbeitslosenquote in durchschnittlichen Gegenden höher sind. Und auch die Wahlbeteiligung unterscheidet sich: 62 Prozent der Befragten aus „sehr schlechter“, „schlechter“ oder „durchschnittlicher" Wohnumgebung geben an, bei der Bundestagswahl 2009 gewählt zu haben, während die Werte für "gute“ und „sehr gute“ Wohngegenden bei 82 bzw. 87 Prozent liegen. ${ }^{15}$ Diese aus Individualdaten ermittelten Werte stimmen mit den oben präsentierten Zusammenhängen auf Stadtteilebene überein, geben aber noch

15 Für Chicago findet schon Tingsten (1975 [1937], S. 157-158) ähnliche Muster: In schlechten Wohngegenden liegt die Wahlbeteiligung deutlich niedriger als in guten. 
keinen Aufschluss darüber, ob Unterschiede in der Wahlbeteiligung durch überindividuelle Faktoren verursacht werden.

Tabelle 4: Wahlbeteiligung Bundestagswahl 2009

\begin{tabular}{|c|c|c|c|}
\hline AV: habe gewählt (1=ja; $0=$ nein $)$ & Modell 1 & Modell 2 & Modell 3 \\
\hline Alter & $\begin{array}{l}0,045^{* *} \\
(0,017)\end{array}$ & $\begin{array}{c}0,019 \\
(0,021)\end{array}$ & $\begin{array}{l}-0,004 \\
(0,021)\end{array}$ \\
\hline Alter $^{2}$ & $\begin{array}{l}-0,000 \\
(0,000)\end{array}$ & $\begin{array}{c}0,000 \\
(0,000)\end{array}$ & $\begin{array}{c}0,000 \\
(0,000)\end{array}$ \\
\hline $\begin{array}{l}\text { Geschlecht } \\
\text { (1=weiblich) }\end{array}$ & $\begin{array}{l}-0,201 * \\
(0,101)\end{array}$ & $\begin{array}{l}-0,127 \\
(0,115)\end{array}$ & $\begin{array}{c}0,055 \\
(0,121)\end{array}$ \\
\hline $\begin{array}{l}\text { in Partnerschaft lebend } \\
(1=\mathrm{ja} ; 0=\text { nein })\end{array}$ & $\begin{array}{l}0,347^{* *} \\
(0,115)\end{array}$ & $\begin{array}{c}0,149 \\
(0,130)\end{array}$ & $\begin{array}{c}0,169 \\
(0,135)\end{array}$ \\
\hline $\begin{array}{l}\text { arbeitslos } \\
(1=\text { ja; } 0=\text { nein })\end{array}$ & $\begin{array}{l}-0,604 * * \\
(0,191)\end{array}$ & $\begin{array}{c}0,044 \\
(0,220)\end{array}$ & $\begin{array}{c}0,126 \\
(0,221)\end{array}$ \\
\hline \multicolumn{4}{|c|}{ Wohnumgebung (RK = „schlecht“ bis „durchschnittlich“) } \\
\hline gut & $\begin{array}{l}0,933^{* * *} \\
(0,113)\end{array}$ & $\begin{array}{l}0,716^{* * *} \\
(0,127)\end{array}$ & $\begin{array}{l}0,701 * * * \\
(0,132)\end{array}$ \\
\hline sehr gut & $\begin{array}{l}1,225^{* * *} \\
(0,141)\end{array}$ & $\begin{array}{l}0,900 * * * \\
(0,166)\end{array}$ & $\begin{array}{l}0,859 * * * \\
(0,172)\end{array}$ \\
\hline \multicolumn{4}{|c|}{ Bildung ( $R K=$ böchstens Hauptschule) } \\
\hline mittlere Reife & & $\begin{array}{l}0,532 * * * \\
(0,135)\end{array}$ & $\begin{array}{l}0,362 * * \\
(0,140)\end{array}$ \\
\hline mind. Fachhochschulreife & & $\begin{array}{l}0,979 * * * \\
(0,174)\end{array}$ & $\begin{array}{l}0,550 * * \\
(0,182)\end{array}$ \\
\hline Haushaltseinkommen (logarithmiert) & & $\begin{array}{l}1,035^{* * *} \\
(0,129)\end{array}$ & $\begin{array}{l}0,921 * * * \\
(0,133)\end{array}$ \\
\hline $\begin{array}{l}\text { Gewerkschaftsmitglied } \\
(1=\mathrm{ja} ; 0=\text { nein })\end{array}$ & & & $\begin{array}{c}0,216 \\
(0,195)\end{array}$ \\
\hline $\begin{array}{l}\text { Parteimitglied } \\
(1=\mathrm{ja} ; 0=\text { nein })\end{array}$ & & & $\begin{array}{l}2,460 * \\
(1,042)\end{array}$ \\
\hline $\begin{array}{l}\text { Kirchgang } \\
(1=\text { nie } \rightarrow 5 \text { häufig })\end{array}$ & & & $\begin{array}{l}0,140 * * \\
(0,051)\end{array}$ \\
\hline $\begin{array}{l}\text { politisches Interesse } \\
\text { (1=überhaupt nicht } \rightarrow 5=\text { sehr stark) }\end{array}$ & & & $\begin{array}{l}0,552 * * * \\
(0,067)\end{array}$ \\
\hline Konstante & $\begin{array}{l}-1,146 * * \\
(0,363)\end{array}$ & $\begin{array}{l}-8,400 * * * \\
(0,971)\end{array}$ & $\begin{array}{l}-5,456^{* * *} \\
(1,054)\end{array}$ \\
\hline pseudo $R^{2}$ & 0,087 & 0,149 & 0,202 \\
\hline$N$ & 2.797 & 2.297 & 2.291 \\
\hline
\end{tabular}

Anmerkungen: Standardfehler in Klammern, ${ }^{*} p<, 05,{ }^{* *} p<, 01,{ }^{* * *} p<, 001$.

Quelle: ZA-Nr. 4610, ALLBUS 2010. 
Deshalb wird im nächsten Schritt unter Berücksichtigung der Wohnumgebung die Wahrscheinlichkeit geschätzt, bei der Bundestagswahl 2009 seine Stimme abgegeben zu haben. Im ersten Schritt werden nur wenige demografische Variablen sowie die dreistufige Bewertung der Wohnumgebung in die Analyse eingeschlossen. Dabei zeigt sich, dass die Wohnumgebung einen signifikanten positiven Effekt auf die individuelle Wahlwahrscheinlichkeit hat (Tabelle 4): Je besser die Wohngegend durch den Interviewer bewertet wird, desto höher liegt die Wahlteilnahme der Befragten. Daran ändert sich nichts, wenn erst das Bildungsniveau und das Haushaltseinkommen (Modell 2), dann Gewerkschafts- und Parteimitgliedschaft sowie Kirchgang und politisches Interesse (Modell 3) als zusätzliche erklärende Variablen aufgenommen werden. Substanziell bedeutet dies, dass die individuelle Wahlwahrscheinlichkeit beim Übergang von einer höchstens durchschnittlichen zu einer sehr guten Wohnumgebung um 12 Prozentpunkte steigt - selbst wenn alle anderen Variablen konstant gehalten werden.

Obwohl in diesem Abschnitt zwei unterschiedliche Datensätze verwendet wurden, die die soziale Umwelt der Befragten durch verschiedene Fragen erfassen, ergeben sich dennoch in beiden Fällen Hinweise auf Kontexteffekte: Wenn man in einer höchstens durchschnittlichen Wohngegend oder in einem Viertel mit hohem Nichtwähleranteil wohnt, verringert sich - unabhängig von anderen individuellen Merkmalen - die Bereitschaft, wählen zu gehen. Dies legt nahe, dass soziale Segregation nicht einfach die Nichtwähler ungleich über eine Stadt verteilt, sondern Rückwirkungen auf die Partizipationsbereitschaft der in einem Viertel lebenden Bürger ausübt.

Bisher konnte nachgewiesen werden, dass sich auf Stadtteilebene deutliche Unterschiede in der Wahlbeteiligung zeigen und dass diese nicht ausschließlich auf individuelle Merkmale der Bewohner zurückzuführen sind. Im nächsten Abschnitt wird nun der für diesen Aufsatz zentralen Frage nachgegangen, ob ein Zusammenhang zwischen der Höhe der Wahlbeteiligung und dem Wahlergebnis besteht.

\section{Die Effekte ungleicher Wahlbeteiligung auf die Parteien}

In Abbildung 4 wird für jede im Bundestag vertretene Fraktion der Zusammenhang zwischen der Wahlbeteiligung bei der Bundestagswahl 2009 und dem Parteiergebnis in 1455 Stadtteilen dargestellt. Für die Unionsparteien, die FDP und in geringerem Maß für die Grünen gilt, dass sie dort die besten Ergebnisse erzielen, wo die Wahlbeteiligung am höchsten ist. Dagegen besteht ein negativer Zusammenhang zwischen der Wahlbeteiligung und dem Ergebnis von Sozialdemokraten und Linkspartei. Die SPD ist die einzige Partei, bei der dieser Trend sich zwischen ost- und westdeutschen Großstädten unterscheidet. Während sich in Westdeutschland ein deutlicher negativer Zusammenhang zwischen der Wahlbeteiligung und dem SPD-Ergebnis zeigt, besteht in Ostdeutschland kein Zusammenhang. Für beide Landesteile gilt jedoch: Je niedriger die Wahlbeteiligung, desto besser schneiden 
SPD und Linkspartei in der Addition ab; und je höher die Wahlbeteiligung, umso bessere Ergebnisse erzielt das bürgerliche Lager aus FDP und Unionsparteien. ${ }^{16}$

\section{Abbildung 4: Wahlbeteiligung und Parteiergebnisse in 1495 Stadtteilen von 34 deutschen Großstädten}
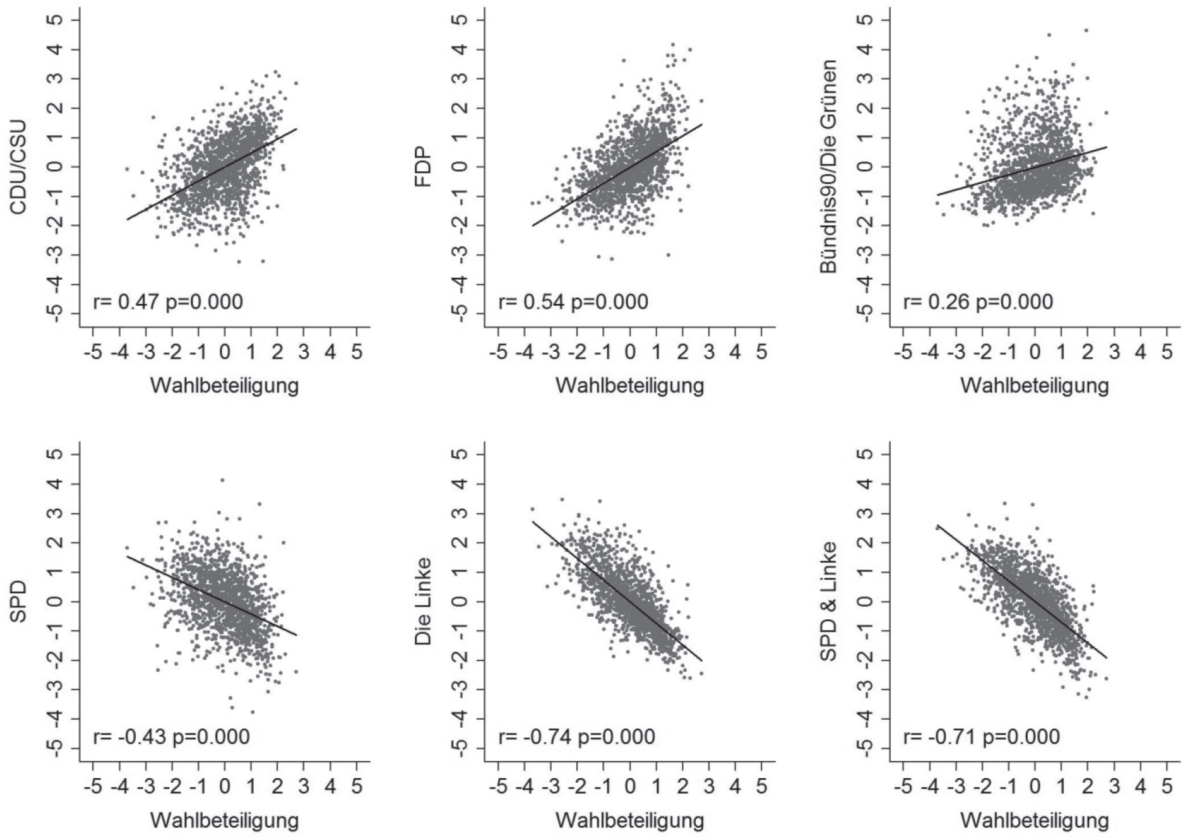

Anmerkung: Die Variablen sind für jede Stadt z-transformiert, sodass der Mittelwert jeweils o und die Standardabweichung 1 beträgt.

Quelle: Eigene Zusammenstellung auf Grundlage der Informationen der städtischen Statistikämter.

Auf den ersten Blick scheint es verblüffend, dass auf Stadtteilebene zwischen der Wahlbeteiligung und dem Abschneiden der Mitte-links-Parteien ein negativer $\mathrm{Zu}-$ sammenhang besteht, wird doch meist angenommen, dass eine höhere Beteiligung diesen Parteien nutzt. Auf aggregierter Ebene gilt dies auch, wie Abbildung 5 am Beispiel Bremens verdeutlicht: Je höher die Wahlbeteiligung bei einer Bundestagswahl in der Stadt Bremen ist, desto besser schneidet die SPD ab. ${ }^{17}$ Im Zeitverlauf sinkt die Wahlbeteiligung und die Ergebnisse der Sozialdemokratie verschlechtern

16 Da Bündnis 90/Die Grünen häufig in sozial heterogenen Innenstädten gute Ergebnisse erzielen, sind sie räumlich weder dem einen noch dem anderen Lager zuzuordnen. Das zeigt sich auch daran, dass einzig bei den Grünen kein substanzieller Zusammenhang zwischen der sozialen Lage ei-

nes Stadtteils gemessen ander Arbeitslosenquote - und dem Parteiergebnis besteht.

17 Bremen ist meines Wissens die einzige Großstadt, für die für einen so langen Zeitraum kleinräumige Wahldaten vorliegen. Häufig werden Daten auf Stadtteilebene erst seit einigen Jahren erhoben oder der Zuschnitt der Stadtteile ändert sich im Zeitverlauf, sodass die Werte nicht vergleichbar sind. Um die Ergebnisse abzusichern, analysiere ich unten zusätzlich Wahldaten aus Köln und Duisburg für etwas kürzere Zeiträume. 
sich. Warum dies so ist, macht der Blick auf Abbildung 6 klar: Auch heute noch schneiden SPD und CDU bei Bundestagswahlen in ihren früheren Hochburgen überdurchschnittlich gut ab. Zwar ist der Zusammenhang für die CDU etwas schwächer, aber dennoch lässt sich für beide Parteien sagen, dass sie nach fast vierzig Jahren noch immer in denselben Vierteln besonders gute oder schlechte Ergebnisse einfahren. Zwei Veränderungen sind allerdings bemerkenswert. Zum einen liegt das beste SPD-Ergebnis 200936 Prozentpunkte niedriger als 1972. Für die CDU ist die Differenz nur halb so groß. Zum anderen korreliert das SPD-Ergebnis von 1972 negativ mit der Veränderung der Wahlbeteiligung zwischen 1972 und 2009, während die Korrelation bei der CDU positiv ist. Das heißt, in SPDHochburgen ist die Wahlbeteiligung systematisch stärker zurückgegangen als in Vierteln mit hohem CDU-Wähleranteil. ${ }^{18}$

Abbildung 5: Wahlbeteiligung in der Stadt Bremen und SPD-Stimmenanteil

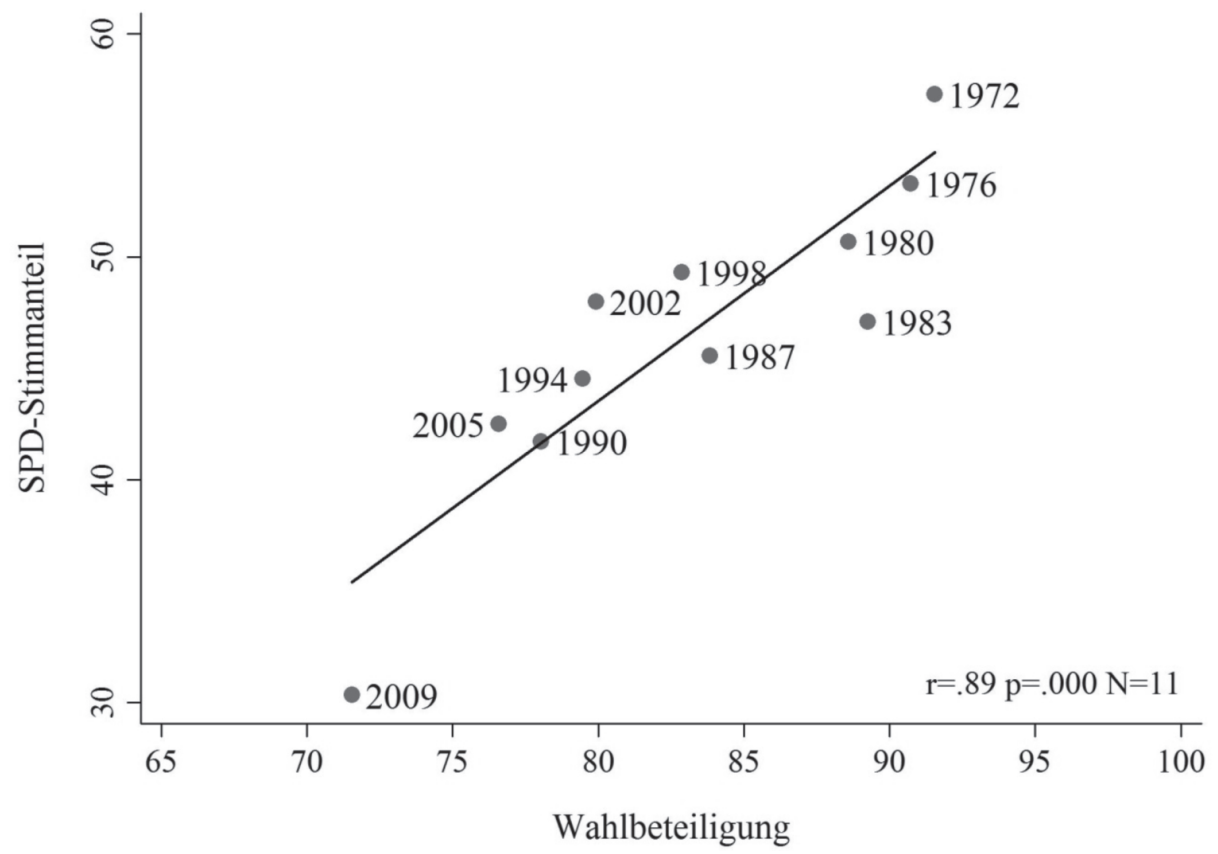

Quelle: Statistisches Landesamt Bremen.

18 Dasselbe Muster zeigt sich in Köln: Die räumliche Verteilung der Parteihochburgen ist seit 1987 weitgehend stabil geblieben. Während der Rückgang der Wahlbeteiligung jedoch systematisch SPD-Hochburgen betrifft, besteht kein Zusammenhang zum CDU-Ergebnis. 
Abbildung 6: Veränderung der Wahlbeteiligung in SPD- und CDU-Hochburgen in Bremen
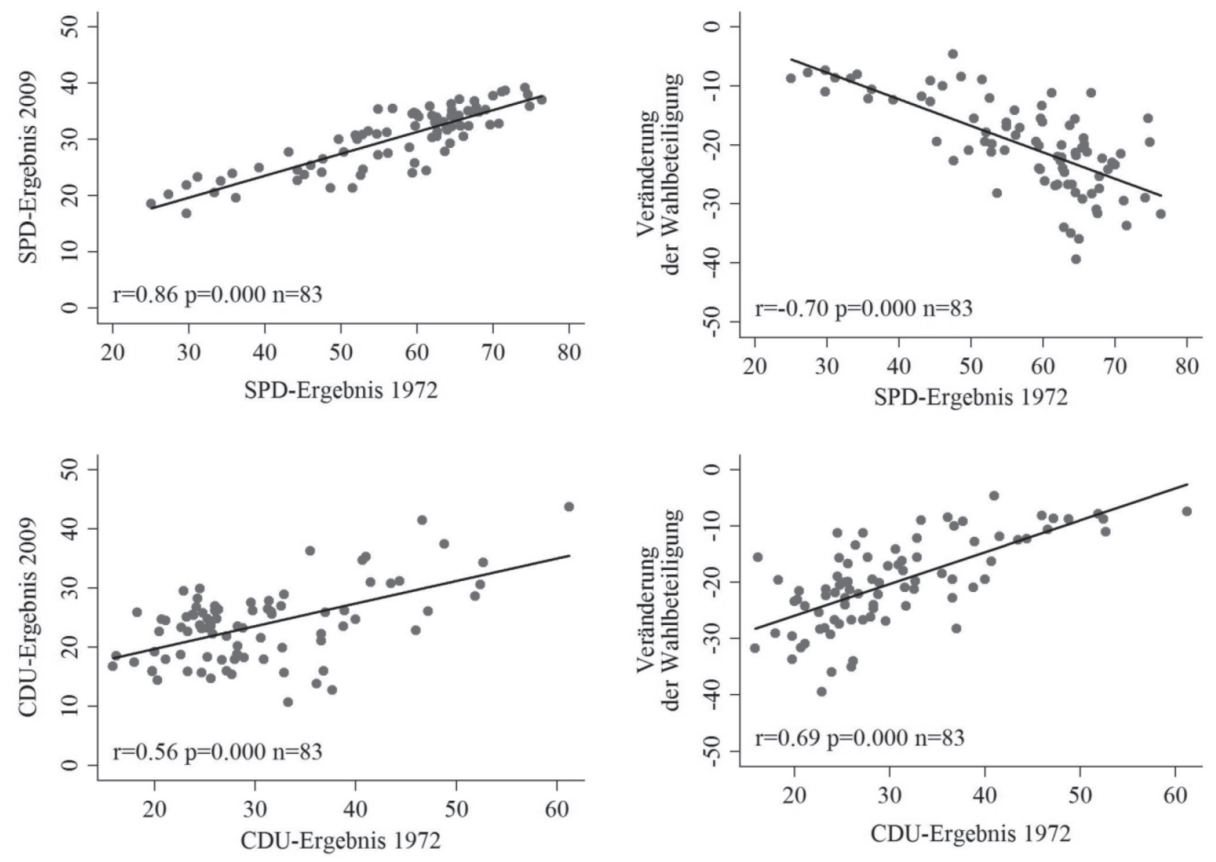

Quelle: Statistisches Landesamt Bremen.

Wie dieser Veränderungsprozess abgelaufen ist, lässt sich aus Abbildung 7 ablesen. Dargestellt ist, wie sich der Zusammenhang zwischen der Wahlbeteiligung in den 83 Bremer Stadtteilen und dem SPD-Ergebnis bei allen Bundestagswahlen seit 1972 entwickelt hat. Korrelierten beide Faktoren anfangs nicht, ist der Zusammenhang im Zeitverlauf stärker geworden. Spätestens seit den 1990ern schneidet die SPD in Bremen in jenen Stadtteilen am besten ab, wo die Wahlbeteiligung niedrig ist. Für die Gesamtstadt ergibt sich deshalb ein positiver Zusammenhang zwischen der Wahlbeteiligung und dem SPD-Ergebnis, weil in der Vergangenheit die Wahlbeteiligung in allen Stadtteilen hoch war. Seither ist sie jedoch asymmetrisch gefallen. Bei der heute niedrigen Wahlbeteiligung treten erhebliche Unterschiede zwischen den Stadtteilen auf - und damit entsteht auf kleinräumiger Ebene ein negativer Zusammenhang zwischen der Wahlbeteiligung und dem Abschneiden von Mitte-links-Parteien. 
Abbildung 7: Wahlbeteiligung und SPD-Ergebnis in 83 Bremer Stadtteilen, 1972-2009
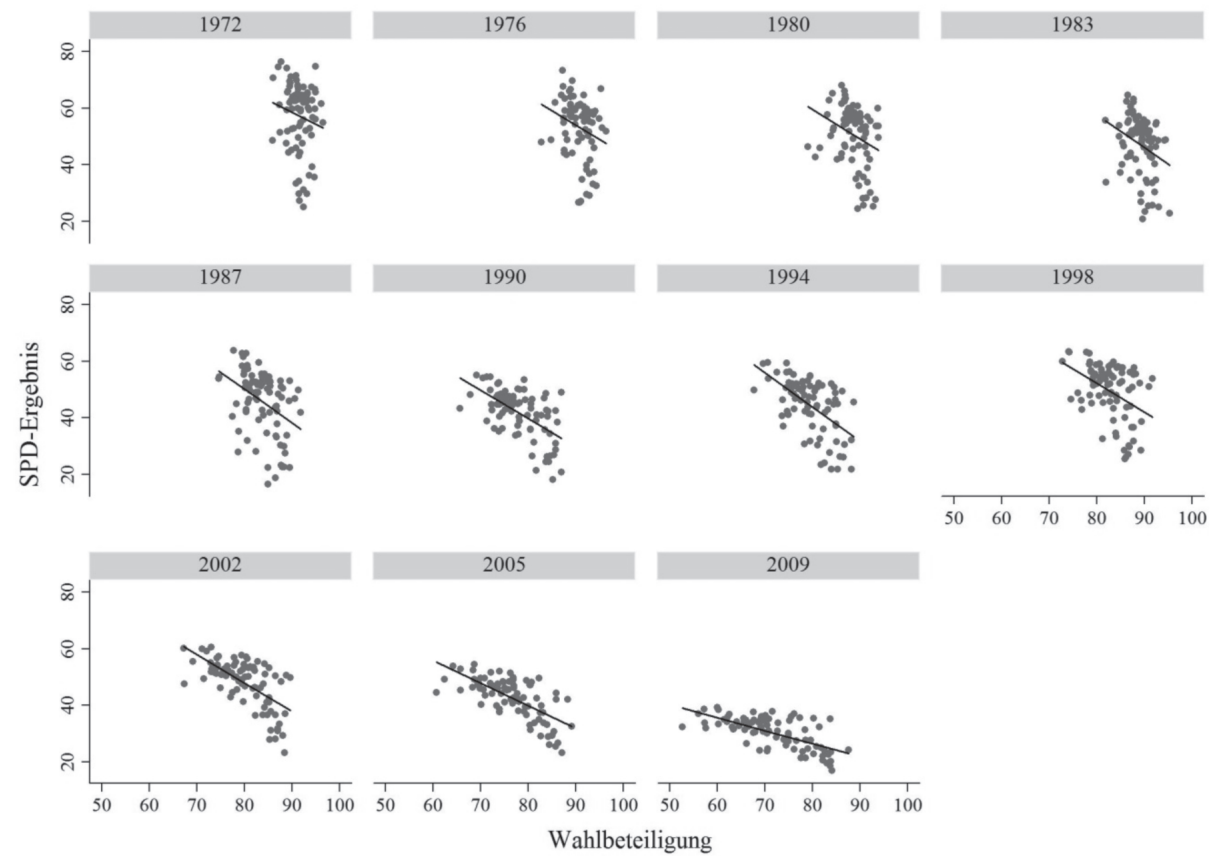

Quelle: Statistisches Landesamt Bremen.

Nachdem der Blick auf deskriptive Statistiken nahelegt, dass ein Zusammenhang zwischen der rückläufigen Wahlbeteiligung und dem Wahlergebnissen besteht, soll geprüft werden, wie sich Veränderungen der Wahlbeteiligung in den Bremer Stadtteilen auf Veränderungen des SPD-Stimmenanteils auswirken. Mit dieser Vorgehensweise soll ausgeschlossen werden, dass nur ein Scheinzusammenhang zwischen beiden Merkmalen besteht, der auf parallelen, aber unabhängigen Trends beruht. ${ }^{19}$ In Tabelle 5 sind die Ergebnisse verschiedener Modellspezifikationen abgetragen, die jedoch ausnahmslos auf einen positiven Effekt der Wahlbeteiligung auf das SPD-Ergebnis hinweisen: Nimmt die Wahlbeteiligung um einen Prozent zu, steigt der SPD-Stimmenanteil um 0,75 Prozent. ${ }^{20}$ Der Zusammenhang besteht auch dann, wenn für das Aufkommen von Bündnis90/Die Grünen und Linkspartei kontrolliert

19 Ich folge hier dem Vorgehen Fishers (2007), um auszuschließen, dass abweichende Ergebnisse durch die Methode zustande kommen. In weiteren, nicht aufgeführten Modellen wurde getestet, ob Autokorrelation (und panel-spezifische Autokorrelation) die Ergebnisse beeinträchtigt. Aber in allen Modellspezifikationen blieb der Effekt der Wahlbeteiligung erhalten.

20 Dieser Zusammenhang ist unabhängig von der Wahl des Untersuchungszeitraums. Auch für die Jahre 1972-1998, 1980-2009 und 1980-1998 ist der Koeffizient für die Wahlbeteiligungsänderung signifikant und positiv, sodass das Ergebnis nicht von einzelnen Wahlen abhängt, in denen die Wahlbeteiligung besonders hoch (oder niedrig) ausfällt und die SPD besonders gut (oder schlecht) abschneidet. 
wird. Zugewinne der Linkspartei wirken sich zwar negativ auf das SPD-Ergebnis aus - allerdings zusätzlich zum Effekt der Wahlbeteiligung. Das heißt, in den letzten Jahren hat die SPD in den Bremer Ortsteilen nicht nur unter der rückläufigen Wahlbeteiligung gelitten, sondern auch Stimmen an die Linkspartei verloren. Addiert man die Ergebnisse der beiden Parteien, zeigt sich ebenfalls ein positiver Zusammenhang zwischen einer Wahlbeteiligungszunahme und der Veränderung ihres Stimmenanteils. Führt man die Analysen aus Tabelle 5 für die anderen Parteien durch, zeigen sich nur für die FDP signifikante Effekte. ${ }^{21}$ Für die Freien Demokraten ist der Effekt der Wahlbeteiligung jedoch gegenläufig: Sinkt die Wahlbeteiligung um einen Prozent, verbessert sich das Wahlergebnis um 0,65 Prozent.

Die bisher präsentierten Ergebnisse widersprechen der vorherrschenden Auffassung, dass eine sinkende Wahlbeteiligung keinen Effekt auf das Wahlergebnis hat. Um diesen kontroversen Befund abzusichern, führe ich exakt dieselbe Analyse für zwei weitere Städte durch, für die über längere Zeiträume Daten auf Stadtteilebene verfügbar sind. Tabelle 6 enthält für Duisburg und Köln die Koeffizienten, wie sich die Veränderung der Wahlbeteiligung auf das SPD-Ergebnis auswirkt. Aus Platzgründen werden die Kontrollvariablen nicht dargestellt. Die Ergebnisse bestätigen für beide Städte, dass der Rückgang der Wahlbeteiligung in einem Stadtteil mit einem Rückgang des SPD-Ergebnisses einhergeht - unabhängig davon, welches Modell geschätzt wird. In der Summe wecken die empirischen Ergebnisse dieses Aufsatzes Zweifel an der These, dass die Höhe der Wahlbeteiligung keine Auswirkungen auf das Abschneiden der Parteien hat. Manche Effekte zeigen sich nur, wenn kleinräumige Daten analysiert werden, bei denen die Folgen der ungleichen Wahlbereitschaft sozialer Gruppen klarer zum Ausdruck kommen.

Tabelle 5: Veränderung des SPD-Stimmenanteils in Bremer Stadtteilen in Abhängigkeit von der Wahlbeteiligung, 1972-2009

\begin{tabular}{|c|c|c|c|c|c|c|}
\hline $\begin{array}{l}\text { AV: Veränderung des } \\
\text { SPD-Stimmenanteils }\end{array}$ & OLS $^{1}$ & $\begin{array}{l}\text { Fixed } \\
\text { Effects }^{1}\end{array}$ & PCSE & $\begin{array}{l}\text { Arellano } \\
\& \text { Bond }\end{array}$ & PCSE & PCSE \\
\hline $\begin{array}{l}\text { Veränderung der } \\
\text { Wahlbeteiligung }\end{array}$ & $\begin{array}{l}0,780 * * * \\
(0,044)\end{array}$ & $\begin{array}{l}0,791 * * * \\
(0,045)\end{array}$ & $\begin{array}{l}0,780 * * \\
(0,264)\end{array}$ & $\begin{array}{l}0,793 * * * \\
(0,046)\end{array}$ & $\begin{array}{l}0,754 * * \\
(0,263)\end{array}$ & $\begin{array}{c}0,795 * * * \\
(0,225)\end{array}$ \\
\hline $\begin{array}{l}\text { Veränderung } \\
\text { SPD-Anteil (lagged) }\end{array}$ & & & & $\begin{array}{l}0,228 * * * \\
(0,042)\end{array}$ & & \\
\hline Zeittrend & & & & & $\begin{array}{c}-0,203 \\
0,361)\end{array}$ & \\
\hline $\begin{array}{l}\text { Veränderung } \\
\text { Bündnis 90/Die Grünen }\end{array}$ & & & & & & $\begin{array}{l}-0,409 \\
(0,295)\end{array}$ \\
\hline $\begin{array}{l}\text { Veränderung } \\
\text { Die Linke }\end{array}$ & & & & & & $\begin{array}{r}-1,132 * * * \\
(0,276))\end{array}$ \\
\hline $\mathrm{R}^{2}$ & 0,293 & 0,293 & 0,293 & - & 0,306 & 0,752 \\
\hline $\mathrm{N}$ & 830 & 830 & 830 & 664 & 830 & 415 \\
\hline
\end{tabular}

1) Modelle mit geclusterten Standardfehlern. Anmerkungen: ${ }^{*} \mathrm{p}<, 05,{ }^{* *} \mathrm{p}<, 01,{ }^{* * *} \mathrm{p}<, 001$.

Quelle: Statistisches Landesamt Bremen. Standardfehler in Klammern.

21 Diese Analysen werden aus Platzgründen hier nicht widergegeben. 
Tabelle 6: Veränderung des SPD-Stimmenanteils in Duisburger und Kölner Stadtteilen in Abhängigkeit von der Wahlbeteiligung

\begin{tabular}{lcccccc}
\hline Duisburg (1990-2009) & OLS $^{1}$ & $\begin{array}{c}\text { Fixed } \\
\text { Effects }^{1}\end{array}$ & PCSE & $\begin{array}{c}\text { Arellano } \\
\text { \& Bond }\end{array}$ & PCSE & PCSE \\
\hline Veränderung der & $1,050 * * *$ & $1,066 * * *$ & $1,050 * *$ & $0,994 * * *$ & $0,598^{* * *}$ & $0,772 * * *$ \\
Wahlbeteiligung & $(0,035)$ & $(0,037)$ & $(0,136)$ & $(0,060)$ & $(0,211)$ & $(0,178)$ \\
$\mathrm{R}^{2}$ & 0,799 & 0,812 & 0,799 & & 0,853 & 0,753 \\
$\mathrm{~N}$ & 230 & 230 & 230 & 138 & 230 & 138 \\
\hline Köln (1987-2009) & & & & & & \\
\hline Veränderung der & $0,652 * * *$ & $0,663 * *$ & $0,652 *$ & $0,937 * *$ & $0,461 *$ & $0,550 *$ \\
Wahlbeteiligung & $(0,031)$ & $(0,031)$ & $(0,323)$ & $(0,057)$ & $(0,206)$ & $(0,239)$ \\
$\mathrm{R}^{2}$ & 0,297 & 0,299 & 0,297 & & 0,644 & 0,607 \\
$\mathrm{~N}$ & 509 & 509 & 509 & 338 & 509 & 256 \\
\hline
\end{tabular}

1) Modelle mit geclusterten Standardfehlern. Anmerkungen: Standardfehler in Klammern; ${ }^{*} \mathrm{p}<, 05,{ }^{* *}$ $\mathrm{p}<, 01,{ }^{* * *} \mathrm{p}<, 001$.

Quelle: Amt für Stadtentwicklung und Statistik der Stadt Köln sowie Stabsstelle für Wahlen, Europaangelegenheiten und Informationslogistik der Stadt Duisburg.

\section{Zusammenfassung und Ausblick}

In diesem Aufsatz bin ich der Frage nachgegangen, ob der Rückgang der Wahlbeteiligung einen Einfluss auf die Wahlergebnisse hat. Entgegen der in der Literatur vorherrschenden Meinung zeigt die Analyse von kleinräumigen Daten aus deutschen Großstädten, dass linke Parteien schlechter abschneiden, wenn die Wahlbeteiligung sinkt. Der Grund liegt in der ungleichen räumlichen Verteilung der Nichtwähler: In „städtischen Problemgebieten “ - häufig frühere Arbeiterviertel und SPD-Hochburgen - ist die Wahlbeteiligung seit den 1970er-Jahren sehr viel stärker gesunken als in von Arbeitslosigkeit und Armut weniger betroffenen Vierteln, sodass heute ein enger $\mathrm{Zu}$ sammenhang zwischen der sozialen Lage eines Stadtteils und der Wahlbeteiligung sowie dem Wahlergebnis besteht. Je ärmer ein Stadtteil, desto niedriger fällt die Wahlbeteiligung aus und desto besser schneiden Parteien links der Mitte ab, während umgekehrt gilt, dass die CDU und vor allem die FDP ihre besten Ergebnisse dort erzielen, wo noch annähernd das Beteiligungsniveau der Vergangenheit erreicht wird.

Die empirischen Ergebnisse dieses Aufsatzes widersprechen der Auffassung, dass die Höhe der Wahlbeteiligung für den Wahlausgang unerheblich und daher demokratietheoretisch nicht entscheidend ist. Sie führen darüber hinaus zu weiteren Forschungsfragen, die verdeutlichen, dass der Blick auf die kleinräumige Ebene von Stadtteilen für die politikwissenschaftliche Forschung fruchtbar ist:

- Lassen sich die Ergebnisse der Aggregatdatenanalyse mit Hilfe von Individualdaten untermauern? Dabei wäre der Frage nachzugehen, ob sich die in Abschnitt 5 skizzierte Ansteckungsgefahr der Nichtwahl erhärten lässt. Beeinflusst die räumliche Konzentration von Nichtwähler das Wahlverhalten der in einem Vierteln Lebenden? Über welche Mechanismen geschieht dies? 
- Wie reagieren die Parteien auf Unterschiede in der Wahlbeteiligung? Vermehren Mitte-links-Parteien die Mobilisierungsanstrengungen in armen Stadtvierteln, in denen sie relativ gut abschneiden, oder konzentrieren sich die Parteien auf Gegenden, in denen besonders viele Wähler anzutreffen sind, wie dies die Literatur zum „,rational prospecting “ annimmt (Brady et al. 1999)?

- Welche Folgen hat die ungleiche Wahlbeteiligung auf politische Entscheidungen? Haben die Anliegen von Bewohnern armer dieselbe Chance wie die wohlhabender Stadtteile, von der Politik berücksichtigt zu werden? Lassen sich, im Umkehrschluss, unpopuläre Schritte auch in Stadtteilen durchsetzen, in denen Bürger mit guter Organisations- und Konfliktfähigkeit beheimatet sind?

Hinter Angaben zur durchschnittlichen Wahlbeteiligung verbergen sich Unterschiede, die erst durch die Analyse kleinräumiger Daten deutlich werden. Die Effekte einer sinkenden Wahlbeteiligung sind gravierender, so das Fazit dieses Aufsatzes, als dies in der Forschung bislang angenommen wird.

\section{Literatur}

Berinsky, Adam J. 2002. Silent Voices: Social Welfare Policy Opinions and Political Equality in America. American Journal of Political Science 46:276-287.

Bernstein, Robert, Anita Chadha und Robert Montjoy. 2001. Overreporting Voting. Why It Happens and Why It Matters. Public Opinion Quarterly 65:22-44.

Brady, Henry E., Kay Lehmann Schlozman und Sydney Verba. 1999. Prospecting for Participants: Rational Expectations and the Recruitment of Political Activists. American Political Science Review 93:153-168.

Caballero, Claudio. 2005. Nichtwahl. In Handbuch Wablforschung, Hrsg. Jürgen W. Falter und Harald Schoen, 329-365. Wiesbaden: VS Verlag für Sozialwissenschaften.

Citrin, Jack, Eric Schickler und John Sides. 2003. What if Everyone Voted? Simulating the Impact of Increased Turnout in Senate Elections. American Journal of Political Science 47:75-90.

Clausen, Aage R. 1968-69. Response Validity: Vote Report. Public Opinion Quarterly 43:588-606.

Eijk, Cees van der, Hermann Schmitt und Eliyahu V. Sapir. 2010. Die politischen Konsequenzen der niedrigen Wahlbeteiligung bei der Europawahl 2009. Politische Vierteljahresschrift 51:605-617.

Eilfort, Michael. 1994. Die Nichtwähler: Wablenthaltung als Form des Wäblerverhaltens. Paderborn: Ferdinand Schöningh.

Erikson, Robert S. 1995. State Turnout and Presidential Voting: A Closer Look. American Politics Research 23:387-396.

Falter, Jürgen W., und Siegfried Schumann. 1993. Nichtwahl und Protestwahl: Zwei Seiten einer Medaille. Aus Politik und Zeitgeschichte 43:36-49.

Falter, Jürgen W., und Siegfried Schumann. 1994. Der Nichtwähler - das unbekannte Wesen. In Wähler und Nichtwähler. Analysen aus Anlaß der Bundestagswabl 1990, Hrsg. Hans-Dieter Klingemann und Max Kaase, 161-213. Opladen: Westdeutscher Verlag. 
Farwick, Andreas. 2007. Die räumliche Polarisierung von Armut in der Stadt - Ursachen, Ausprägungen und soziale Folgen. In Armutsbericht 2007, Hrsg. Arbeiterkammer Bremen, 73-87. Bremen: Arbeiterkammer Bremen.

Fisher, Stephen D. 2007. (Change in) Turnout and (Change in) the Left Share of the Vote. In Electoral Studies 26:598-611.

Friedrichs, Jürgen, und Sascha Triemer. 2008. Gespaltene Städte? Soziale und ethnische Segregation in deutschen Großstädten. Wiesbaden: VS Verlag für Sozialwissenschaften.

Gardemin, Daniel. 2009. Kleinräumige Untersuchung der SPD-Wählerschaft - Wahlanalysen in Hannover von 1998 bis 2008. In Die Krise der SPD. Autoritäre oder partizipatorische Demokratie, Hrsg. Heiko Geiling, 259-299. Münster u. a.: LIT Verlag.

Geiling, Heiko. 2009. Die SPD im freien Fall - Zwischenrufe zu den niedersächsischen Landtagswahlen 2003 und 2008. In Die Krise der SPD. Autoritäre oder partizipatorische Demokratie, Hrsg. Heiko Geiling, 213-257. Münster u. a.: LIT Verlag.

Hajnal, Zoltan L. 2010. America's Uneven Democracy. Race, Turnout, and Representation in City Politics. Cambridge: Cambridge University Press.

Hajnal, Zoltan, und Jessica Trounstine. 2005. Where Turnout Matters: The Consequences of Uneven Turnout in City Politics. Journal of Politics 67:515-535.

Hansford, Thomas G., und Brad T. Gomez. 2010. Estimating the Electoral Effects of Voter Turnout. American Political Science Review 104:268-2010.

Highton, Benjamin, und Raymond E. Wolfinger. 2001. The Political Implications of Higher Turnout. British Journal of Political Science 31:179-223.

Hennig, Eike, Robert Lohde-Reiff und Detlef Sack. 2001. Wahlenthaltung in der Großstadt: Das Beispiel Frankfurt am Main. Frankfurter Statistische Berichte 3:224251.

Jagodzinski, Wolfgang, Jürgen Friedrichs und Hermann Dülmer. 1996. Bestimmungsgründe der Wahlbeteiligung und der Wahl rechtsextremistischer Parteien - Ergebnisse von Aggregatdatenanalysen zweier deutscher Großstädte. In Wablen und politischer Einstellungen in westlichen Demokratien, Hrsg. Oscar W. Gabriel und Jürgen W. Falter, 119-147. Frankfurt a. M.: Peter Lang.

Kleinhenz, Thomas. 1995. Die Nichtwähler. Ursachen der sinkende Wablbeteiligung in Deutschland. Opladen: Westdeutscher Verlag.

Kohler, Ulrich. 2006. Die soziale Ungleichheit der Wahlabstinenz in Europa. In Europas Osterweiterung: Das Ende der Vertiefung? WZB-Jahrbuch 2005, Hrsg. Jens Alber und Wolfgang Merkel, 159-179. Berlin: edition sigma.

Kohler, Ulrich. 2011. Estimating the Potential Impact of Nonvoters on Outcomes of Parliamentary Elections in Proportional Systems with an Application to German National Elections from 1949 to 2009. Electoral Studies 30:497-509.

Lavies, Ralf-Rainer. 1973. Nichtwählen als Kategorie des Wablverhaltens. Empirische Untersuchung zur Wablenthaltung in historischer, politischer und statistischer Sicht. Düsseldorf: Droste Verlag.

Lijphart, Arend. 1997. Unequal Participation: Democracy's Unresolved Dilemma. American Political Science Review 91:1-14.

Lutz, Georg, und Michael Marsh. 2007. Introduction: Consequences of Low Turnout. Electoral Studies 26:539-547. 
Mahler, Vincent A. 2008. Electoral Turnout and Income Redistribution by the State: A Cross-National Analysis of the Developed Democracies. European Journal of Political Research 47:161-183.

Pacek, Alexander, und Benjamin Radcliff. 1995. Turnout and the Vote for Left-of-Centre Parties: A Cross-National Analysis. British Journal of Political Science 25:137-143.

Pappi, Franz Urban. 1977. Sozialstruktur und politische Konflikte in der Bundesrepublik. Individual- und Kontextanalysen der Wahlentscheidung. Köln: Universität zu Köln.

Radcliff, Benjamin. 1994. Turnout and the Democratic Vote. American Politics Quarterly 22:259-276.

Robinson, William S. 1950. Ecological Correlation and the Behavior of Individuals. American Sociological Review 15:351-357.

Schäfer, Armin. 2011a. Der Nichtwähler als Durschnittsbürger: Ist die sinkende Wahlbeteiligung eine Gefahr für die Demokratie? In Der unbekannte Wähler? Mythen und Fakten über das Wahlverhalten der Deutschen, Hrsg. Evelyn Bytzek und Sigrid Rossteutscher, 133-154. Frankfurt a. M.: Campus Verlag.

Schäfer, Armin. 2011b. Compulsory Voting and Republican Liberty. MPIfG Discussion Paper 11/17. Köln: Max-Planck-Institut für Gesellschaftsforschung.

Schlichting, Karl. 2009. Wahlverhalten in der Stadt Bremen nach ausgewählten Gebietstypen. In Bericht zur sozialen Lage 2009. Schwerpunkt: Wablverdrossenheit, Hrsg. Arbeiterkammer Bremen, 46-63. Bremen: Arbeiterkammer Bremen.

Schmitt-Beck, Rüdiger, Christian Mackenrodt und Thorsten Faas. 2008. Hintergründe kommunaler Wahlbeteiligung. Eine Fallstduie zur Kommunalwahl 2004 in Duisburg. Zeitschrift für Parlamentsfragen 39:561-580.

Schoen, Harald. 2005. Daten in der empirischen Wahlforschung. In Handbuch Wablforschung, Hrsg. Jürgen W. Falter und Harald Schoen, 89-103. Wiesbaden: VS Verlag für Sozialwissenschaften.

Schoof, Peter. 1981. Wahlbeteiligung und Sozialstruktur in der Bundesrepublik. Eine Aggregatdatenanalyse für den Zeitraum von 1972 bis 1980. Politische Vierteljahresschrift 22:287-304.

Steinbrecher, Markus, Sandra Huber und Hans Rattinger. 2007. Turnout in Germany. Citizen Participation in State, Federal, and European Elections since 1979. Baden-Baden: Nomos.

Tourangeau, Roger, Robert M. Groves und Cleo D. Redline. 2010. Sensitive Topics and Reluctant Respondents. Demostrating a Link Between Nonresonse Bias and Measurement Error. Public Opinion Quarterly 74:413-432.

Tingsten, Herbert. 1975 [1937]. Political Behavior. Studies in Election Statistics. London: Arno Press.

\section{Autorenangaben:}

Dr. Armin Schäfer,

Max-Planck-Institut für Gesellschaftsforschung (MPIfG), Paulstraße 3, 50676 Köln, schaefer@mpifg.de 